\title{
Radiación solar en entornos urbanos forestados con clima seco. \\ Caso: Área Metropolitana de Mendoza, Argentina.
}

Solar radiation in forested urban environments with dry climate.

Case: Metropolitan Area of Mendoza, Argentina.

Dra. Mariela Arboit, Dr. Ernesto Betman

\section{Filiación}

Consejo Nacional de Investigaciones Científicas y Técnicas, Argentina.

E mail: marboit@lab.cricyt.edu.ar

Primera versión recibida en: 11 de noviembre de 2013

Última versión recibida en: 19 de diciembre de 2014

\section{Resumen}

El objetivo del trabajo es producir avances en el conocimiento del potencial solar de entornos urbanos residenciales los que, por su morfología, y por la incidencia del arbolado urbano, presentan valores de irradiancia muy diferentes a las de pleno asoleamiento. Las variables morfológicas de las configuraciones urbano-edilicias y la arboleda urbana, una característica distintiva del Área Metropolitana de Mendoza (AMM), tienen una incidencia fundamental de primer orden en la factibilidad de implementar estrategias de aprovechamiento de la energía solar en medios urbanos. Los resultados alcanzados permitirán a futuro reformar y actualizar en forma progresiva los códigos urbanos y edilicios para implementar los máximos niveles de eficiencia energética y los mínimos impactos ambientales de las edificaciones urbanas.

Este estudio propone la determinación del potencial de captación solar en ambientes urbanos considerando las variables morfológicas urbano-edilicias correspondientes a 11 configuraciones urbanas representativas del AMM. Metodológicamente se ha monitoreado la irradiancia solar global sobre el plano vertical en fachadas norte completamente asoleadas y parcialmente asoleadas afectadas por enmascaramiento sólido y enmascaramiento del arbolado. Los resultados obtenidos indican que el enmascaramiento solar es crítico en el caso de las superficies verticales con una reducción de la energía solar disponible entre el $2 \%$ y $66 \%$ en la estación de invierno, dependiendo del tipo de arbolado y de la morfología edilicia. Sin embargo, a pesar de esta limitación que puede ser compensada mediante mejoras en aspectos de conservación energética, los beneficios de la arboleda urbana en la estación cálida, son múltiples (Brager, et. al. 2001): control de la intensidad de la isla de calor urbana, absorción de contaminantes, refrescamiento y humectación del aire por evapotranspiración, reducción de las cargas térmicas de los edificios, habitabilidad de los espacios públicos abiertos y un aporte inestimable a la estética urbana.

\section{Palabras claves}

Desarrollo urbano sostenible; Morfología urbana; Potencial solar.

\begin{abstract}
The aim of this work is to advance the understanding of the solar potential of urban residential environments which, by their morphology, and the impact of urban trees, present values of irradiance very different from full solar collection. Morphological variables of urban settings and urban trees, a very distinctive feature of the Mendoza Metropolitan Area (MMA), have a fundamental impact on the feasibility of implementing strategies for solar energy utilization in urban environments. The results achieved will contribute to modify and gradually update urban and building legislation to implement higher levels of energy efficiency and minimum environmental impacts.
\end{abstract}


This work proposes to study the potential of solar collection in urban environments, analyzing eleven urban configurations selected according to their building and urban morphological characteristics.

Methodologically, we have monitored the global solar irradiance on vertical plane on northern facades, completely sunny and partly sunny, affected by solid masking and arboreal masking. Results obtained so far indicate that solar masking is critical for vertical surfaces, with a reduction of the available solar energy between $2 \%$ and $66 \%$ in the winter season. However, these drawbacks caused by urban trees are compensated by benefits in the warm season: controlling the intensity of the urban heat island, absorption of pollutants, cooling and humidifying the air through evapotranspiration, reducing thermal loads of buildings, occupancy of public open spaces, and an invaluable contribution to the urban aesthetic.

\section{Key words}

Sustainable urban development; Urban Morphology; Solar potential.

\section{Sumario}

Introducción

1 Metodología

Los cuatro parámetros son: (Fig. 1)

1.1 Selección de Casos de Estudio

2 Análisis de los resultados

2.1 Influencia de la morfología edilicia heterogénea

2.2 Influencia del ancho de cañón urbano

2.3 Influencia del arbolado

\subsection{INFLUENCIA DE LA ALTURA DE LA EDIFICACION}

\section{Conclusiones}

Bibliografía

\section{Introducción}

El trabajo que se presenta estudia la radiación solar disponible en fachadas Norte en entornos urbanos altamente forestados característicos del Área Metropolitana de Mendoza (AMM) como parte de la problemática del desarrollo ambiental y energéticamente sostenible del sector edilicio en ciudades de la región mesotermal árida sub-andina del centro-oeste de Argentina.

El Área Metropolitana de Mendoza, es el conglomerado urbano más importante de la región, situado en el piedemonte andino, a 750 m.s.n.m., dentro del denominado Oasis Norte. Presenta inviernos templado - fríos, con valores entre $1300^{\circ} \mathrm{C}$ y $1500^{\circ} \mathrm{C}$ día anuales de calefacción, contando con un generoso recurso solar $(4.58 \mathrm{Kwh} / \mathrm{m} 2$ día a $5.55 \mathrm{Kwh} / \mathrm{m} 2)$ y con predominio de cielos claros que aportan una viabilidad comprobada para el uso de la radiación solar para calefacción de espacios y calentamiento de agua mediante sistemas pasivos y colectores planos, respectivamente. (Servicio Meteorológico Nacional y Red Solarimétrica de la República Argentina)

En cuanto a la relevancia de la relación entre el hábitat y la energía, la insostenibilidad del desarrollo actual agravado por el hecho de que la situación se deteriora inexorablemente en el tiempo por falta de conocimientos y de acciones de planificación y gestión. Algunas cifras globales son suficientemente elocuentes para calificarla: a nivel mundial, el hábitat, es decir el conjunto de ciudades y edificios de todo el mundo, consume el $50 \%$ del total de la energía utilizada, 
quedando el $50 \%$ restante dividido en partes aproximadamente iguales entre el transporte y la industria ${ }^{1}$.

Ante este panorama es sumamente interesante aprovechar los recursos naturales y en particular la optimización del acceso al recurso solar para disminuir el consumo de energía no renovable ${ }^{2}$. Ante esta inquietud, surge la pregunta: ¿Cuál es la incidencia de las morfologías urbano-edilicias y de las distintas especies arbóreas que predominan en el entorno urbano en la disponibilidad del recurso de energía solar?

En el AMM se han desarrollado varios estudios específicos a partir de conjuntos representativos de la morfología urbano- edilicia determinado el potencial solar de entornos de baja y alta densidad altamente arbolados y la evolución previsible; con el objetivo de: i. preservar la fisonomía de la ciudad arbolada, manteniendo la escala y la homogeneidad de las construcciones y el aporte estético y los servicios ambientales de la arboleda urbana. ii. Posibilitar el uso máximo del recurso solar para calefacción de espacios y calentamiento de agua sanitaria, mediante el control de la morfología urbana y de la arboleda. iii. Mejorar las condiciones de habitabilidad del parque edilicio. iv. Contribuir a la sostenibilidad del desarrollo local posibilitando el reciclaje de las construcciones existentes de buena calidad y mantenimiento, reduciendo de esta forma los procesos extractivos y la emisión de desechos sólidos (escombros) al ecosistema.

Para completar estos estudios se realizó la medición de irradiancia solar en Fachadas Norte en 11 casos seleccionados de acuerdo a los resultados anteriores. Se pretende realizar una contribución, tanto conceptual como operativa, que por vía de la transferencia permita a futuro que el sector oficial tome conciencia de la gravedad de la situación y comience prontamente a actualizar e implementar nuevas normativas edilicias y urbanas que además apunten a reducir los consumos de gas natural y otras energías no-renovables en los edificios urbanos.

\section{Metodología}

En trabajos previos ${ }^{3}$ se definió el indicador Factor de Asoleamiento efectivo (FAE), que permite analizar la radiación total incidente en fachadas norte en entornos urbanos forestados. El Factor de Asoleamiento Efectivo describe y relaciona la energía disponible con el total de la energía incidente. Expresa la relación entre la sumatoria de la radiación incidente sobre áreas de fachadas orientadas al Norte $\left(+/-15^{\circ}\right)$, parcial y totalmente asoleadas, considerando enmascaramientos sólidos (edificios) y permeables (árboles), para cada hora del día-tipo mensual de cálculo, durante un ciclo anual de calefacción y la sumatoria de la radiación incidente sobre las mismas áreas, libres de todo enmascaramiento, durante el mismo periodo, como porcentaje. EI FAE proporciona una medida del enmascaramiento de superficies potencialmente colectoras para calefacción de espacios, en función de las variables de la morfología urbana consideradas. Se calcula mediante la Ecuación 1 en donde es necesario ingresar los valores de cuatro parámetros.

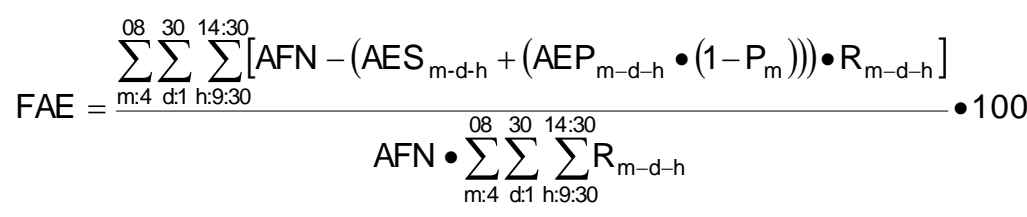

$$
\begin{aligned}
& \text { Ecuación } 1 \\
& \text { Fte: Elaboración propia }
\end{aligned}
$$

${ }^{1}$ DE ROSA, 1988

2 CAPELUTO et. al, 2003; JENKS, 1996; OWENS S. 1986; TOWN AND COUNTRY PLANNING ASSOCIATION, 1996

${ }^{3}$ ARBOIT et. al, 2008; 2010 
Los cuatro parámetros son: (Fig. 1)

- AFN: Área potencialmente colectora total en fachadas norte (m2)

- AES: Área de enmascaramiento sólido (construcciones): fachada potencialmente colectora afectada por las sombras proyectadas por construcciones edilicias próximas (m2). (Figura)

- AEP: Área de enmascaramiento permeable (árboles): fachada potencialmente colectora afectada por las sombras proyectadas por el arbolado urbano.

- Pm: Factor de Permeabilidad mensual. Porcentajes de permeabilidad solar de cada especie vegetal y para cada mes de la estación de calefacción (\%).

- $R(m-d-h)$ : Energía por unidad de superficie disponible en fachadas norte para cada hora, día y mes de la estación de calefacción (Wh/m2)

- Sub-índices: m: mes a calefaccionar. Varía entre abril y septiembre, d. día del mes. Varía entre 1 y 30, h: hora. Varía entre 9:00 hs. y 18:00 hs.

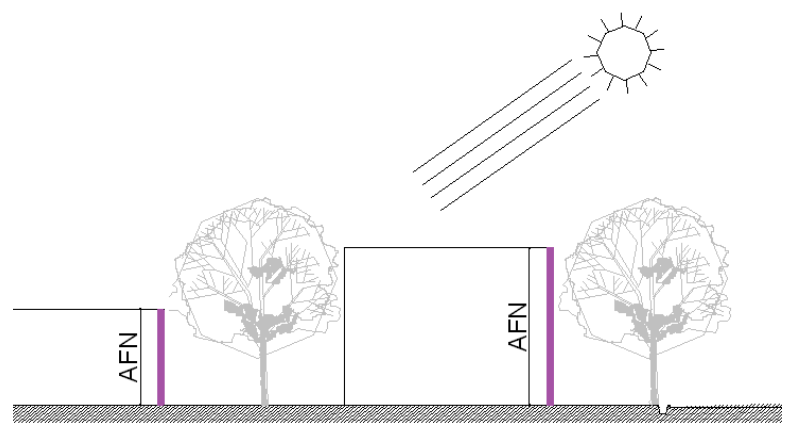

AFN: Área total potencialmente colectora en fachadas norte

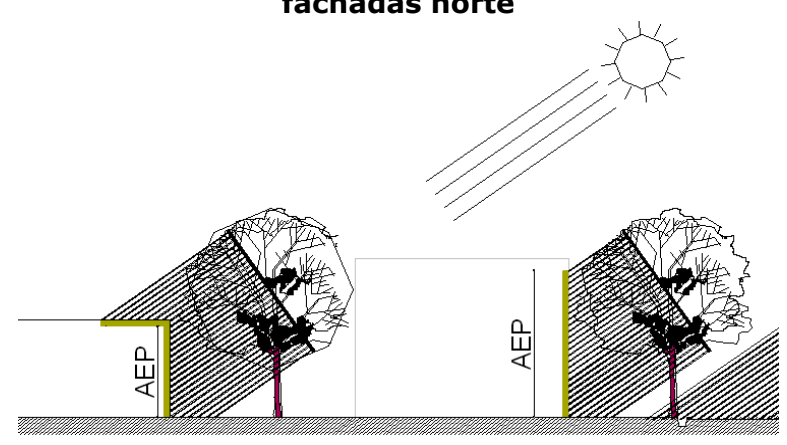

AEP: Área de enmascaramiento permeable (árboles)

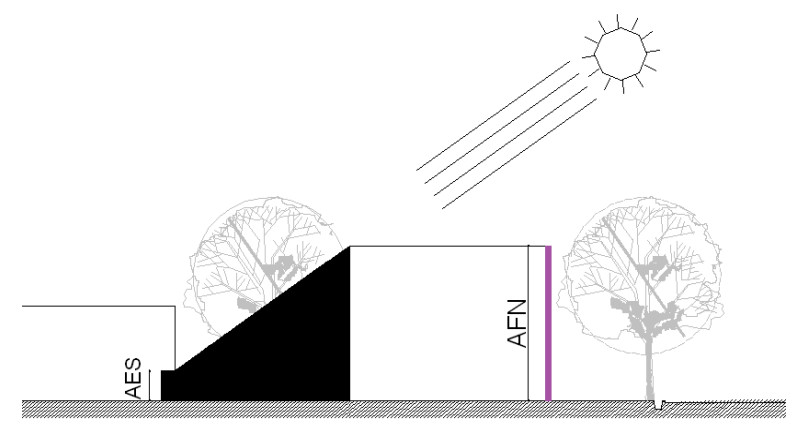

AES: Área de enmascaramiento sólido (construcciones)

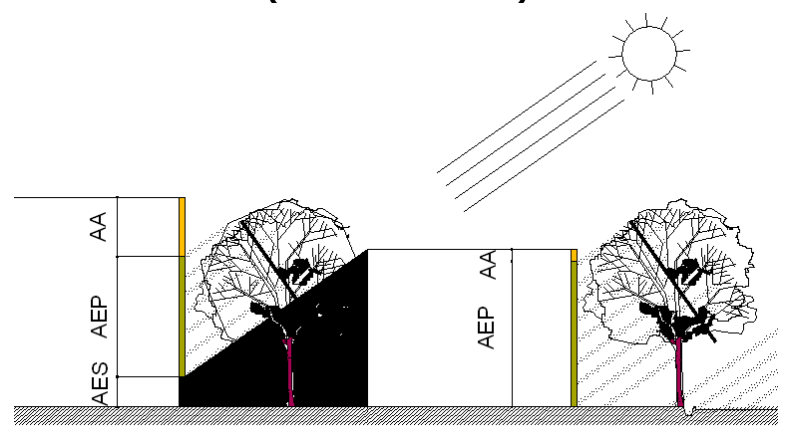

AAN: Área Asoleada Neta: sumatoria de los valores de las superficies asoleadas AA + (AEP * P)(permeabilidad)

Fig. 1 Esquemas descriptivos de la AFN, AES, AEP y AAN Fte: Elaboración propia

Partiendo del Factor de Asoleamiento Efectivo en fachadas norte se determinaron las principales variables que influyen en el acceso al sol ${ }^{4}$. Estas variables son: Morfología Edilicia (Morf.edil), Ancho de Cañón Urbano (An.calles), Arbolado (Plenitud del Arbolado), Altura edilicia (Factor de Ocupación Total -FOT-) utilizando un Modelo Estadístico de Regresión Lineal Múltiple (MERLM), a partir de los valores específicos de cada variable considerada como explicativa, permitiendo además conocer el nivel de incidencia de cada una en el valor resultante. El Factor de

${ }^{4}$ ARBOIT et. al, 2010 
Asoleamiento Efectivo en fachadas norte es el resultado de calcular las variables de mayor impacto mediante la Ecuación 2.

$$
\text { FAEfn }=\frac{\left[\exp ^{(4.1969-0.1486 \text { An. calles }-0.0127 \text { Plen. arb }+0.8547 \text { Morf.edil }-0.4721 \text { FOT })}\right]}{[1+\exp (4.1969-0.1486 \text { An. calles - 0.0127Plen. arb + 0.8547 Morf.edil - 0.4721 FOT })}
$$

\section{Ecuación 2}

Fte: Elaboración propia

Para estudiar la radiación solar hay que considerar que la componente directa incide uniformemente sobre todas las superficies asoleadas de la misma orientación y pendiente, la difusa y la reflejada serán diferentes para cada punto de una superficie dada, teniendo en cuenta la posición relativa del punto considerado con respecto a todos los elementos, edificios y árboles, del campo de vista al $\mathrm{N}$ del plano de fachada que lo contiene. Al considerar una manzana urbana, todos los puntos de cada una de las fachadas deberían tenerse en cuenta, lo cual es claramente imposible por la cantidad de puntos y datos que deberían manejarse. La alternativa que se ha considerado para simplificar la toma de datos es medir la irradiancia solar global sobre el plano vertical de fachada norte completamente asoleado y medir la misma variable sobre la zona de fachada parcialmente asoleada ${ }^{5}$. Esta última está afectada por la morfología urbano-edilicia (morfologías edilicias, ancho de calles, arbolado, altura edilicia).

Las mediciones se realizaron con equipos portátiles de adquisición de datos de irradiancia. Cada equipo está provisto de una unidad de adquisición de datos con un conversor analógico/digital de 12 bits de resolución y una memoria de $64 \mathrm{~K}$ bytes que permite efectuar hasta 43000 mediciones. Como sensor se utilizó un piranómetro weathertronics 3040. Estos sensores tienen un factor de transmisión espectral constante para todas las longitudes de onda comprendidas entre $0.3 \mu \mathrm{m}$ y $60 \mu \mathrm{m}$. Cada piranómetro entrega una diferencia de potencial eléctrico que es proporcional a la irradiancia recibida y que es característica de cada unidad, pero siempre muy cercana a los $15 \mu \mathrm{V} / \mathrm{Wm} 2$. Estos pequeños valores de señal determinan la necesidad de utilizar amplificadores de instrumentación para alcanzar valores que sean compatibles con el rango de tensiones de entrada de la unidad de adquisición de datos (0-2.5V). Como amplificadores de tensión se utilizaron LJTick-InAmp, fabricados por LabJack, USA. La característica más importante de estos amplificadores es que modifican la señal en menos de $0.35 \%$ de la ganancia ideal. Además disponen de varios modos de amplificación que pueden ser seleccionados por el usuario, lo que permite una excelente adaptación al rango de entrada del convertidor A/D de la unidad de adquisición.
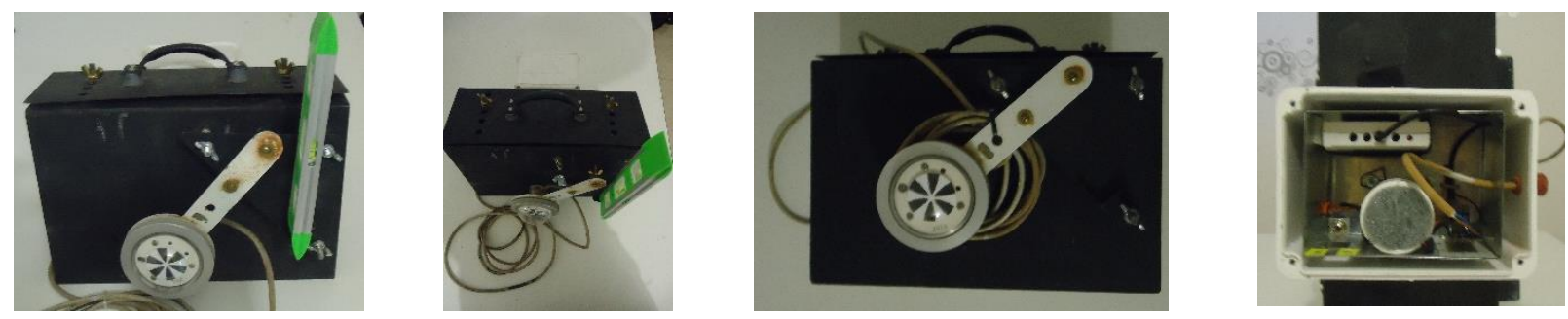

Fig. 2 Equipos portátiles

Fte: Elaboración propia

Se estableció el periodo de medición en función de la latitud y la longitud de la localidad en estudio, para un día dado. Se definió el medio día solar ( $\approx$ 13:30 hora local) y se tomaron cuatro

${ }^{5}$ CÁRDENAS et. al, 2012 
horas y media del periodo comprendido antes del mediodía solar y después del medio día solar. De esta manera quedaron definidas 9 horas solares a medir de 9 a 18hs. Los datos fueron registrados cada 1 minuto durante el mes de julio, agosto y septiembre de 2013. En función de lo planteado, se seleccionaron las mediciones correspondientes a cielo claro para cada entorno urbano; la condición de cielo claro permite evaluar en su completa magnitud la influencia del arbolado y la morfología urbana. Dado que las mediciones se han realizado en el periodo de invierno, se ha evaluado la condición de ramas desnudas del arbolado.
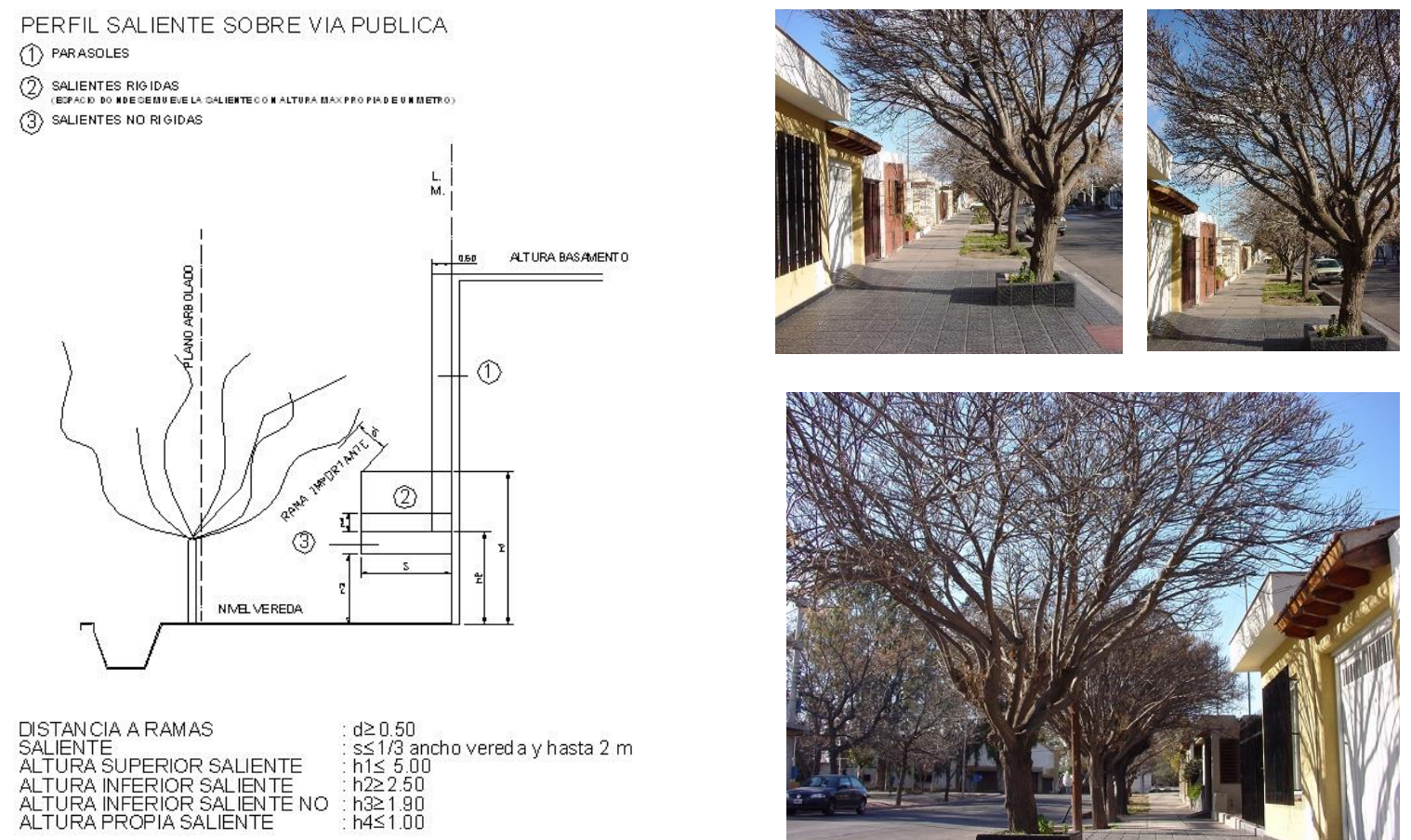

Fig. 3 Entornos urbanos característicos, en condición de ramas desnudas del arbolado. Fte: Código de Edificación. Fotografías: Elaboración propia

\subsection{Selección de Casos de Estudio}

Para el monitoreo de la irradiancia solar global en fachadas norte afectadas por enmascaramiento sólido y enmascaramiento del arbolado se seleccionaron 11 casos de estudio que combinan condiciones de medición distintas: 1. variables urbanas (ancho de cañón urbano, especies arbóreas (magnitud, permeabilidad, distancia de plantación entre individuos) 2. variables edilicias (morfologías edilicias homogéneas, heterogenias, retiros y densidades). Los mismos son representativos de las configuraciones urbanas típicas de la ciudad con perfiles forestados.

Para la selección de los casos de estudio se consideró la morfología urbano-edilicia y forestal de los entornos urbanos del AMM en función del Factor de Asoleamiento Efectivo. Los lineamientos establecidos históricamente en el trazado urbano presentan gran variedad de ancho de calles que van desde 13 metros de ancho hasta los casos que contempla la ley vigente de $20 \mathrm{~m}$ y $30 \mathrm{~m}$. La representatividad de ancho de cañón urbano en el AMM es de: ancho < de 16 metros 23\%, ancho entre 16 y 18 metros $13 \%$, ancho entre 18 y 20 metros $18 \%$, ancho entre 20 y 25 metros $30 \%$ y > de 25 metros $16 \%$.

En el análisis de los entornos urbanos forestados se ha tenido en cuenta la forma, tamaño, permeabilidad solar del arbolado y distancia de plantación entre individuos. El arbolado urbano

${ }^{6}$ ARBOIT, 2008 
público para situaciones típicas del área de Capital, Mendoza, está representada por especies como: Plátano (Platanus acerifolia) $21.80 \%$, Morera (Morus alba) $32.71 \%$, Fresno europeo (Fraxinus excelsior) $20.95 \%$ y Paraíso (Melia azedarach) $2.72 \%{ }^{7}$. En entornos de baja densidad del AMM, las especies son Morera (Morus alba) $41.68 \%$, Fresno europeo (Fraxinus excelsior) $13.05 \%$ y Paraíso (Melia azedarach) $3.58 \%$, existen en urbanizaciones recientes fuertes tendencias de plantación de Acer (Acer negundo) $9.95 \%$ y Paraíso sombrilla (Melia azedarach fm. umbraculifera) $8.37 \%{ }^{8}$.

En la Tabla 1 se observan los casos de estudio y las características de las variables urbanas y edilicias.

\begin{tabular}{|c|c|c|c|c|c|c|c|c|c|}
\hline \multirow{3}{*}{\begin{tabular}{|c} 
Caso \\
$\mathbf{S}$
\end{tabular}} & \multicolumn{6}{|c|}{ VARIABLES URBANAS } & \multicolumn{3}{|c|}{$\begin{array}{l}\text { VARIABLES } \\
\text { EDILICIAS }\end{array}$} \\
\hline & \multicolumn{2}{|c|}{ Manzanas } & \multirow{2}{*}{$\begin{array}{c}\text { Ancho } \\
\text { del } \\
\text { Canal } \\
\text { Vial } \\
(\mathrm{m})\end{array}$} & \multicolumn{3}{|c|}{ Arbolado Urbano } & \multirow[b]{2}{*}{$\begin{array}{c}\text { Morfologí } \\
\text { a }\end{array}$} & \multirow[b]{2}{*}{$\begin{array}{c}\text { Retiros } \\
(\mathrm{m})\end{array}$} & \multirow[b]{2}{*}{$\begin{array}{l}\text { Piso } \\
\text { Nive }\end{array}$} \\
\hline & $\begin{array}{l}\text { Densi- } \\
\text { dad }\end{array}$ & $\mid \begin{array}{c}\text { Orien } \\
- \\
\text { tació } \\
\mathrm{n}\left({ }^{\circ}\right)\end{array}$ & & $\begin{array}{c}\text { Magn } \\
\text { i-tud }\end{array}$ & $\begin{array}{l}\text { Permeabilidad } \\
\text { especies }\end{array}$ & Plenitud & & & \\
\hline 1 & Alta & 13 & 30.00 & $2^{a}$ & Morera(Morus alba) & $\begin{array}{l}\text { Varios } \\
\text { apareados }\end{array}$ & Regular & 0 & $3 e r$ \\
\hline 2 & Baja & 4 & 13.40 & $\begin{array}{l}2^{a} \\
1^{a}\end{array}$ & $\begin{array}{l}\text { Morera(Morus alba) y Fresno } \\
\text { europeo( Fraxinus excelsior } \\
\text { f }\end{array}$ & 2 individuales & Irregular & 5 & $1 \mathrm{er}$ \\
\hline 3 & Alta & 13 & 30.00 & $2^{a}$ & Morera(Morus alba) & $\begin{array}{l}\text { Varios } \\
\text { apareados }\end{array}$ & Regular & 0 & 2do \\
\hline 4 & Baja & 5 & 16.40 & $\begin{array}{l}2^{a} \\
2^{a}\end{array}$ & $\begin{array}{l}\text { Paraíso(Melia azedarach) } \\
\text { Morera(Morus alba) }\end{array}$ & $\begin{array}{l}2 \text { apareados/ E. } \\
\text { jóvenes }\end{array}$ & Regular & 0 & $1 \mathrm{er}$ \\
\hline 5 & Media & 5 & 16.40 & $2^{a}$ & Morera(Morus alba) & Individual & Irregular & 3 & 2do \\
\hline 6 & Alta & 8 & 30.00 & $2^{a}$ & \begin{tabular}{|ll}
$\begin{array}{l}\text { Cipres } \\
\text { sempervirens) }\end{array}$ & (Cupressus \\
\end{tabular} & Individual & Irregular & 2 & $1 \mathrm{er}$ \\
\hline 7 & Media & 7 & 14.20 & $\begin{array}{l}2^{a} \\
1^{a}\end{array}$ & $\begin{array}{l}\text { Morera(Morus alba) } \\
\text { Platano(Platanus acerifolia) }\end{array}$ & $\begin{array}{l}\text { Varios } \\
\text { apareados }\end{array}$ & Irregular & 0 & 2 do \\
\hline 8 & Baja & 29 & 20.88 & $1^{a}$ & Platano(Platanus acerifolia) & Individual/poda & Regular & 0 & $1 \mathrm{er}$ \\
\hline 9 & Baja & 29 & 20.18 & $1^{a}$ & Platano (Platanus acerifolia) & 2 apareados & Irregular & 0 & $1 \mathrm{er}$ \\
\hline 10 & Media & 29 & 20.18 & $1^{a}$ & Platano(Platanus acerifolia) & Individual/poda & Irregular & 0 & 2do \\
\hline 11 & Baja & 29 & 20.88 & $1^{a}$ & Platano(Platanus acerifolia) & 2 apareados & Regular & 0 & 1er \\
\hline
\end{tabular}

Tabla 1 Listado de valores de las variables urbanas y edilicias del conjunto muestral Fte: Elaboración propia

\section{Análisis de los resultados}

La Fig. 4 muestra la diversidad de situaciones energéticas de acuerdo al entorno urbano analizado y marca la preponderancia de cada una de las variables que intervienen en la ecuación 1 . Se observa un porcentaje de reducción del total de la energía solar recibida (enmascaramiento) en las lecturas realizadas de entre el $2 \%$ y el $66 \%$.

\footnotetext{
7 CANTÓN, 2000

${ }^{8}$ ARBOIT, 2010
} 


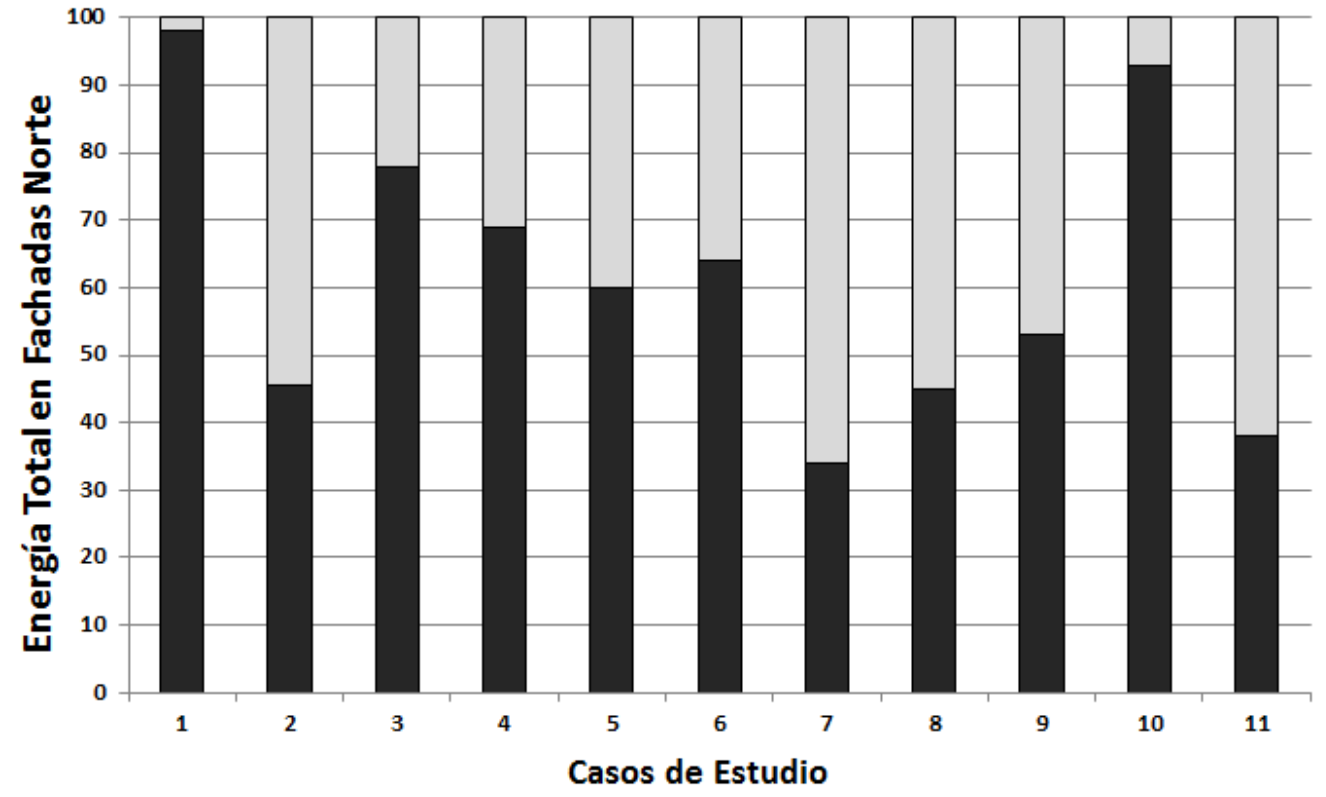

— Energía Neta

$\square$ Enmascaramiento

Fig. 4 Porcentaje Energía Total en Fachadas Norte Wh/m2 Fte: Elaboración propia

Dicho porcentaje depende del entorno urbano edilicio, la reducción del $2 \%$ corresponde al Caso 1 con un cañón urbano de 30mts y morfologías edilicias homogéneas que no presenta superficies sombreadas por los edificios vecinos, además se encuentra a una altura de $10 \mathrm{mts}$ (con escasa influencia del arbolado por altura de la copa). El porcentaje de máxima reducción, del 66\%, corresponde al Caso 7 caracterizado por un ancho de cañón urbano de 14.20 mts, y presenta una importante medida de superficies parcialmente sombreadas por el arbolado de segunda magnitud Morera (Morus alba) y primera magnitud Plátano (Platanus acerifolia), con la consecuente reducción de la energía disponible.

Para un estudio más detallado se han considerado los Casos 1, 2, 3, 6 y 7 que son morfologías urbanas en donde cada una de las variables que intervienen en la ecuación 2 muestra una preponderancia muy definida. De esta manera se analizará cómo influyen en la cantidad de energía solar recibida en fachadas norte las variables: Morfología Edilicia, Ancho de Cañón Urbano, Arbolado y Altura Edilicia.

\subsection{Influencia de la morfología edilicia heterogénea}

El potencial solar de los entornos urbanos seleccionados está determinado por los aspectos de la morfología urbana y edilicia, con las distintas posibilidades de interacción entre la volumetría y el acceso a la radiación solar. En este caso un $64 \%$ de la radiación incidente en la fachada norte es captada, el enmascaramiento se debe principalmente a la morfología edilicia circundante que arroja sombras sobre áreas potencialmente colectoras y al enmascaramiento permeable que presenta el arbolado. 


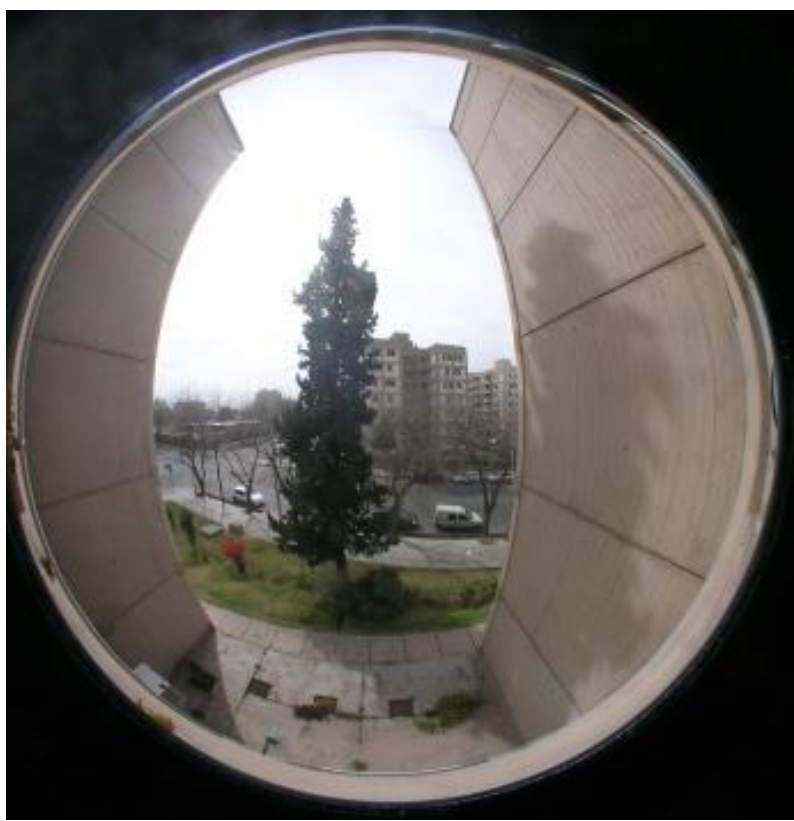

Fig. 5 Fotografía hemisférica del entorno tomada desde la ubicación del sensor Fte: Foto por Lorena Córica

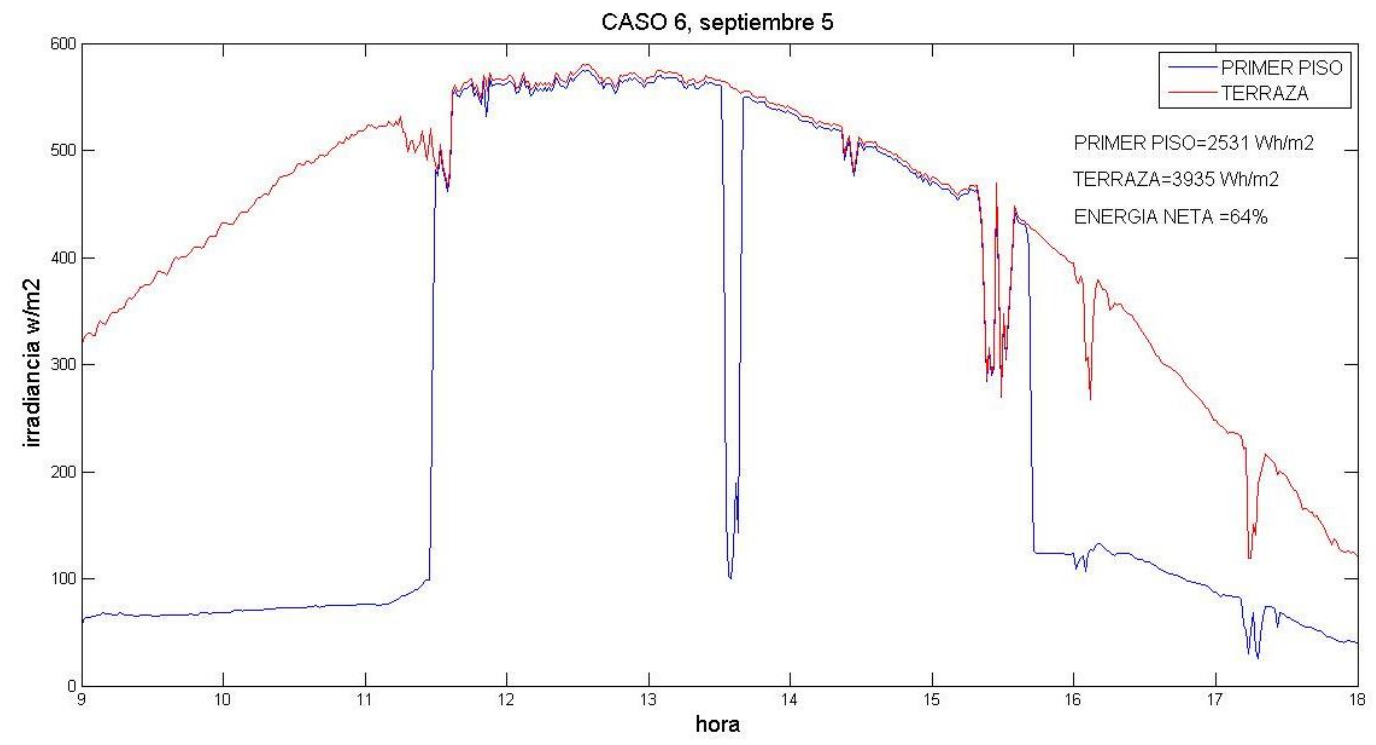

Fig. 6 Irradiancia medida en el plano vertical y orientado hacia el norte a pleno asoleamiento y en condición de asoleamiento parcial

Fte: Elaboración propia 


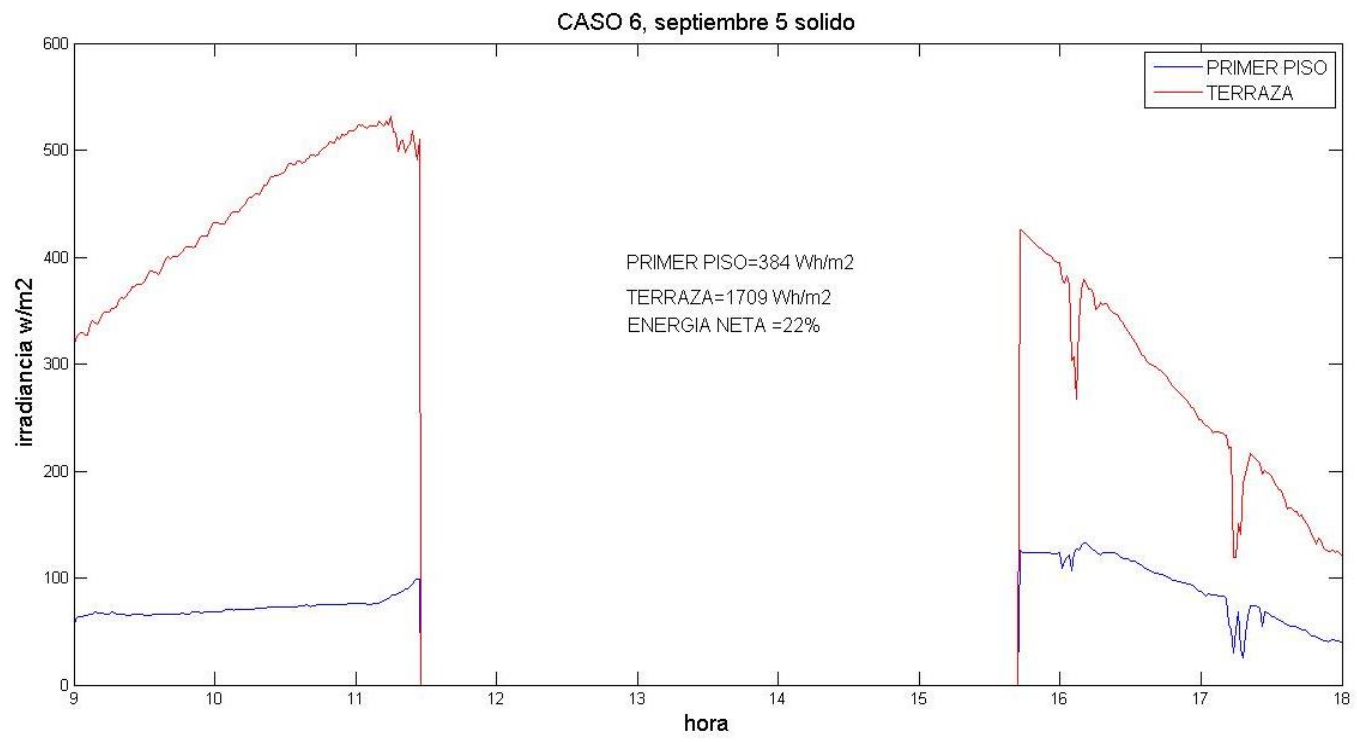

Fig. 7 Irradiancia medida en el plano vertical y orientado hacia el Norte a pleno asoleamiento y en condición de asoleamiento parcial

Fte: Elaboración propia

A los fines de evaluar separadamente el enmascaramiento sólido se ha graficado la Fig. 7.

Hay un acceso a solamente el $22 \%$ de la energía solar disponible que es el aporte de la radiación difusa y reflejada.

Al comparar separadamente la disponibilidad matutina (16\%) y vespertina (34\%) se observa que en el horario de la tarde la radiación reflejada tiene un mayor impacto debido a la desviación de la orientación del trazado urbano respecto al Norte ( $8^{\circ}$ hacia el Este). Debe tenerse en cuenta que la importante reducción del recurso solar que presenta este caso sería mucho mayor si la morfología edilicia afecta el acceso de la radiación solar en las horas centrales del día.

La morfología edilicia ha sido considerada en este análisis en términos la heterogeneidad que genera entrantes y salientes que producen enmascaramiento sólido y comprometen el acceso al sol. Este tipo de morfologías heterogéneas son difícilmente recuperables implementando medidas de reciclaje cuando las condiciones de asoleamiento no se cumplen.

Cuando se planifican y diseñan nuevos conjuntos, la homogeneidad es una condición básica para asegurar el asoleamiento pleno de las superficies potencialmente colectoras, tanto verticales al Norte como horizontales o en pendiente.

\subsection{Influencia del ancho de cañón urbano}

El ancho de los canales viales y su orientación son determinantes del potencial solar de los edificios urbanos, en este caso si bien el sentido e-o posibilita la mejor orientación de la fachada y en consecuencia, su mayor asoleamiento y máxima eficiencia energética, el ancho de canal vial de $14.20 \mathrm{~m}$. compromete seriamente dicho asoleamiento.

Un ancho de cañón urbano estrecho al combinarse con la arboleda urbana de primera magnitud, tiene una incidencia de primer orden en el potencial solar. Los resultados del Caso de Estudio 7 indican una reducción máxima de la energía solar disponible que alcanza un enmascaramiento del $66 \%$ con el ramaje desnudo de sus forestales. 


\section{URBANISMO}

ISSN 0717-5051

http://revistaurbanismo.uchile.cl
Revista de Urbanismo No31 - Julio - Diciembre de 2014 Departamento de Urbanismo - FAU - Universidad de Chile
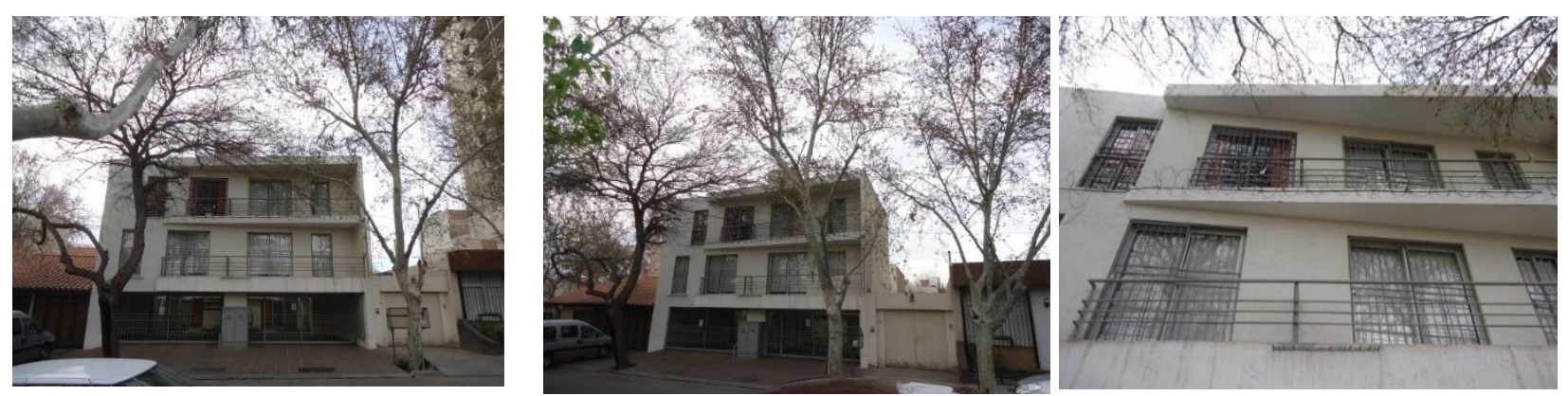

Fig. 8 Vistas de la fachada estudiada. Caso 7 Fte: Elaboración propia

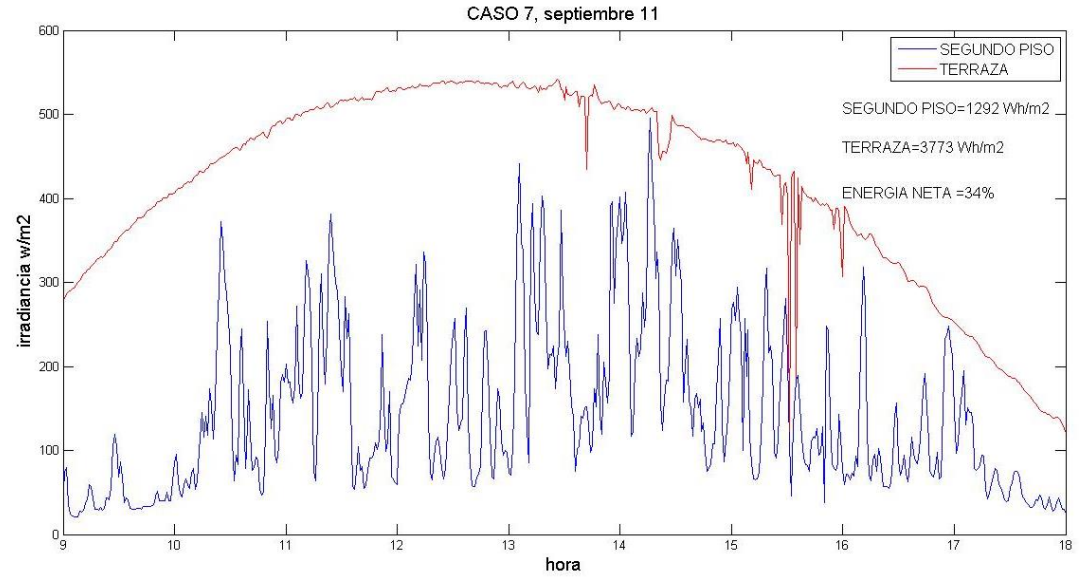

Fig. 9 Irradiancia medida en el plano vertical y orientado hacia el Norte a pleno asoleamiento y en condición de asoleamiento parcial Fte: Elaboración propia

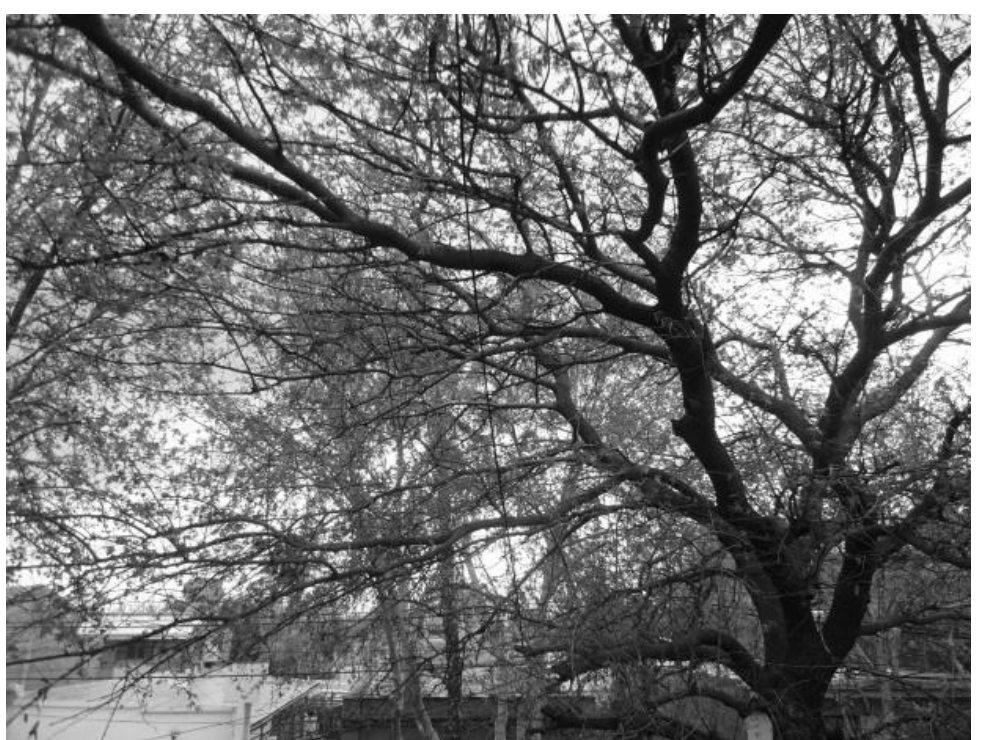

Fig. 10 Fotografía del arbolado desde la ubicación del sensor Fte: Elaboración propia 
El enmascaramiento producido por los ramajes desnudos de los árboles puede ser considerable en invierno, cuando se trata de especies de buen porte $\left(1^{a}\right.$. y $2^{a}$. Magnitud y canales viales angostos (13 a $16 \mathrm{~m}$.).

En este caso se observa que la permeabilidad a la radiación que ofrece el arbolado en canales urbanos estrechos presenta grandes variaciones en función del tiempo y de acuerdo a la posición del sol.

\subsection{Influencia del arbolado}

Las principales variables del arbolado urbano que determinan el acceso al sol son: la magnitud (dependiente de las características morfológicas y dimensionales de la especie arbórea: forma, altura y diámetro de la copa), la permeabilidad estacional del follaje de las especies caducifolias (dependiente de la especie y su ciclo de foliación) a la radiación solar directa y por último, la distancia de plantación entre individuos que en casos genera la superposición de ramajes.

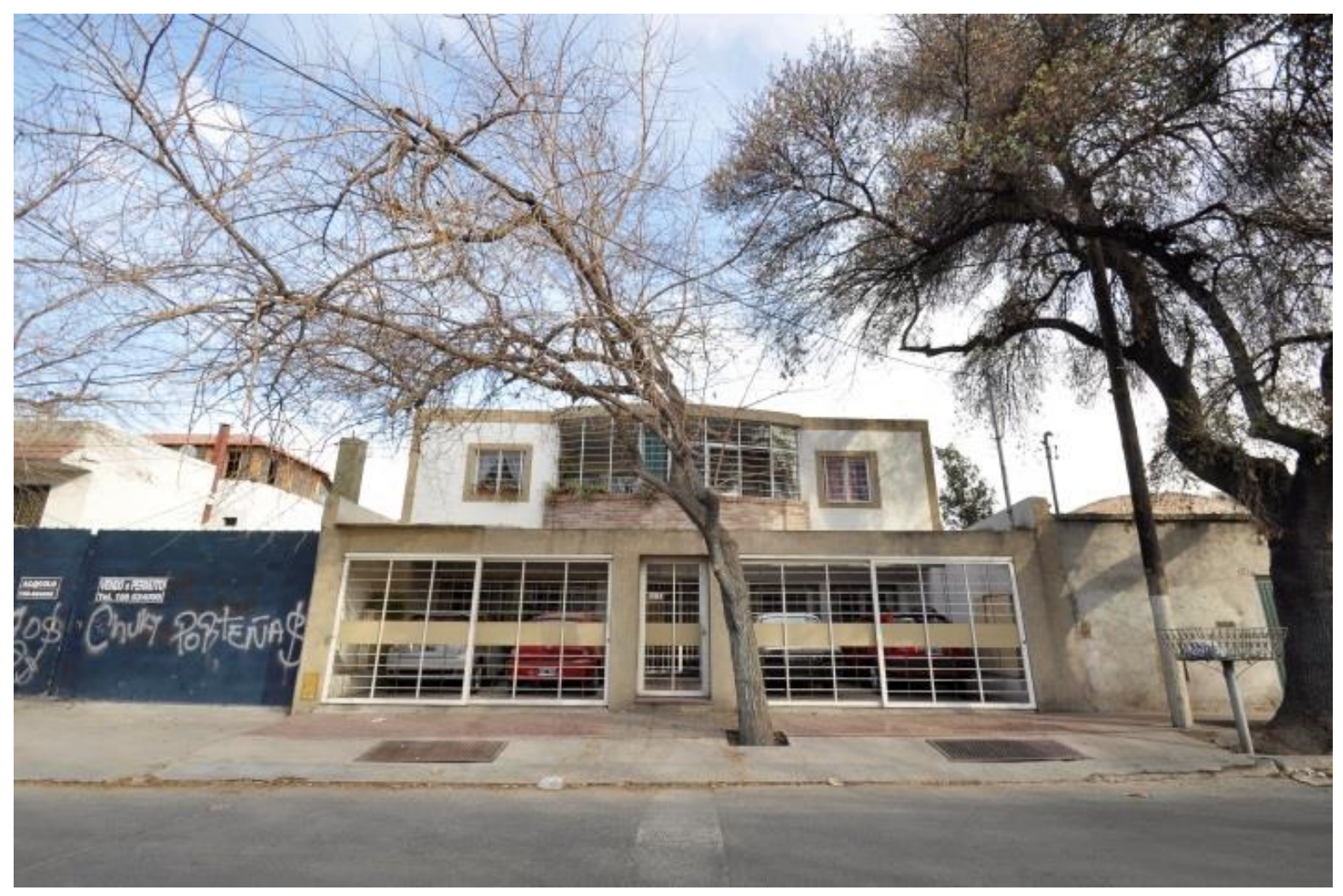

Fig. 11 Fotografía frontal de la fachada

Fte: Elaboración propia 


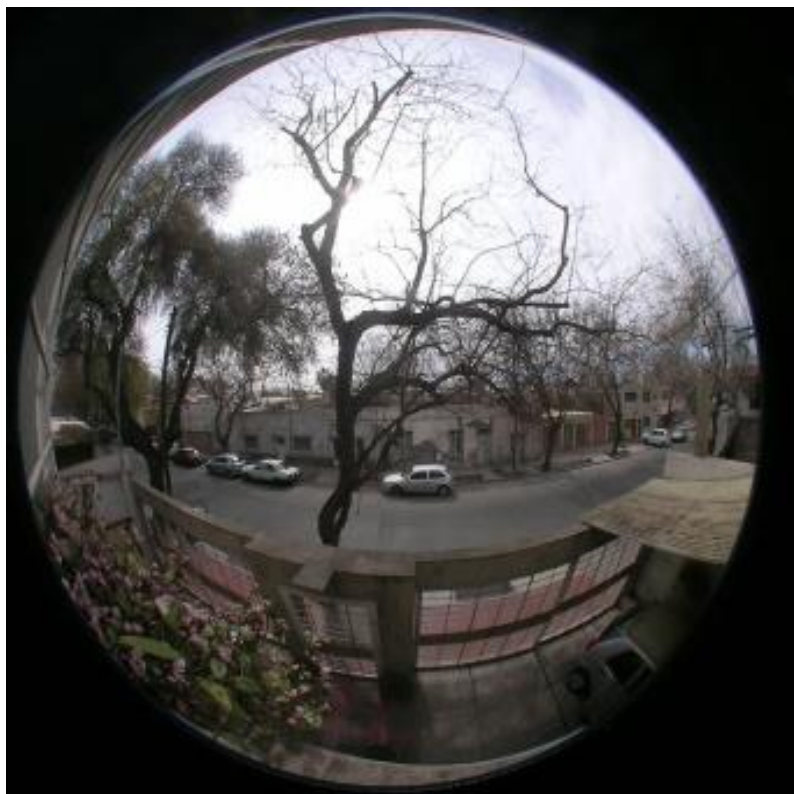

Fig. 12 Fotografía hemisférica del entorno tomada desde la ubicación del sensor Fte: Fotografía por Julieta A. Yamin Garretón

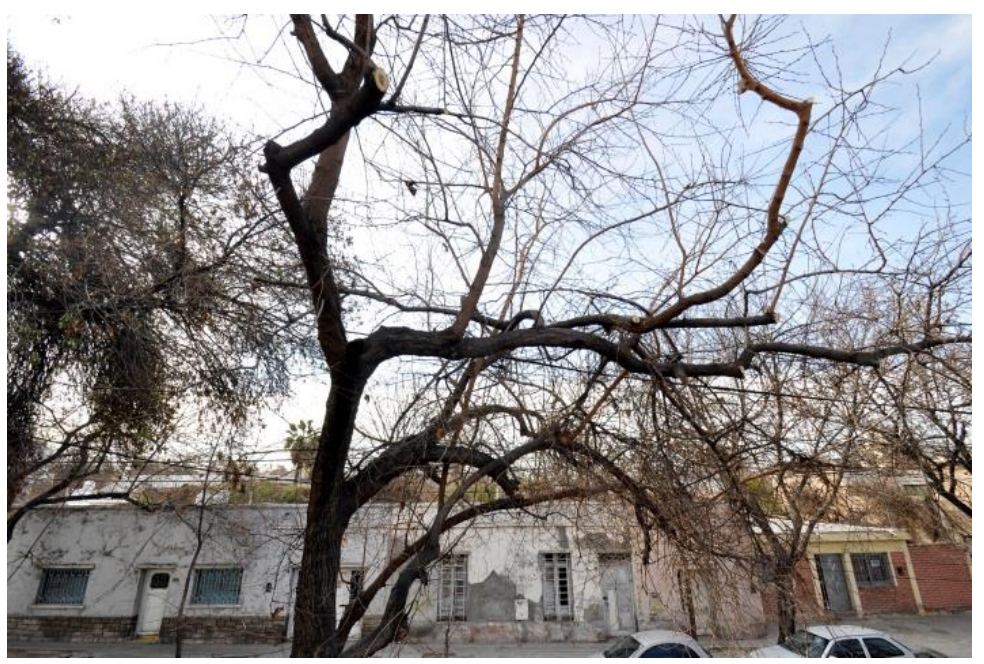

Fig. 13 Fotografía gran angular tomada desde la ubicación del sensor Fte: Elaboración propia

El enmascaramiento en muros orientados al Norte y para los días estudiados es del 55\% (Fig. 14).

En la figura pueden observase la influencia de dos especies diferentes, a los efectos de determinar el impacto de cada una se analizaron separadamente y los resultados obtenidos nos permiten apreciar claramente las diferencias.

En horas de la mañana y centrales del día la disminución en la disponibilidad del recurso solar es del 39\% (Fig. 15) este valor corresponde a la obstrucción que presenta el ramaje desnudo de una Morera (Morus alba) mientras que en horas de la tarde la disminución del recurso es del $85 \%$ debido a la alta densidad de ramaje que presenta el Fresno europeo (Fraxinus excelsior) (Fig. 16). 


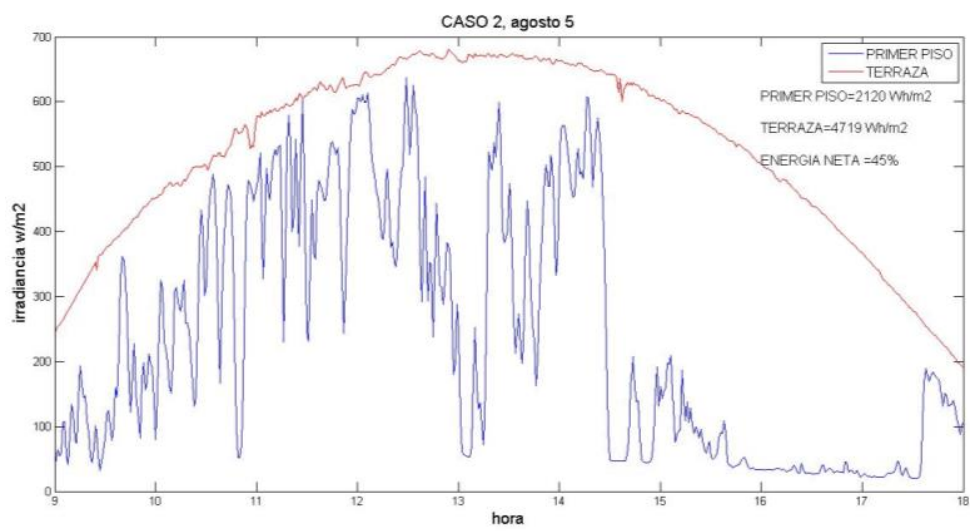

Energía Neta: corresponde al \% de energía que recibe la zona de fachada afectada por la morfología urbana respecto a la energía medida en la zona de fachada libre de obstrucciones.

Fig. 14 Irradiancia medida en el plano vertical y orientado hacia el Norte a pleno asoleamiento y en condición de asoleamiento parcial

Fte: Elaboración propia

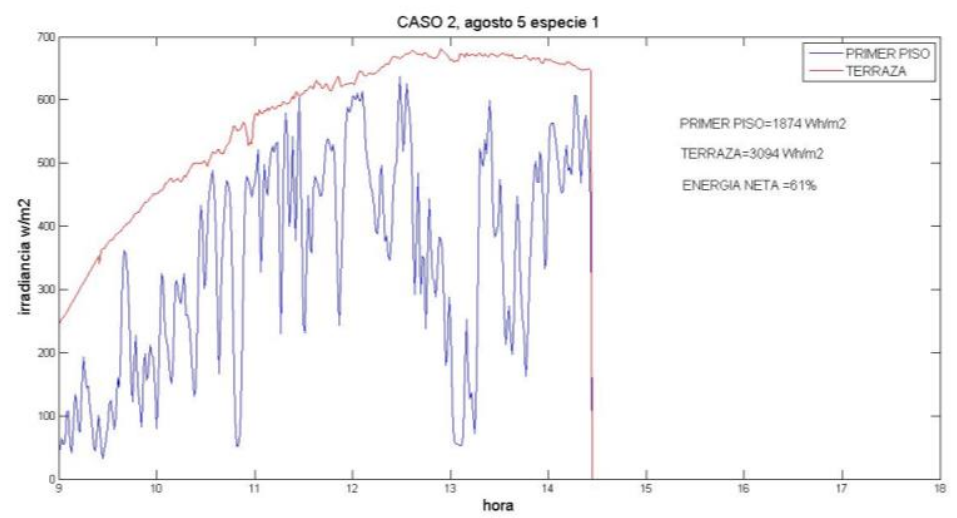

Fig. 15 Irradiancia medida en el plano vertical y orientado hacia el Norte a pleno asoleamiento y en condición de asoleamiento parcial

Fte: Elaboración propia

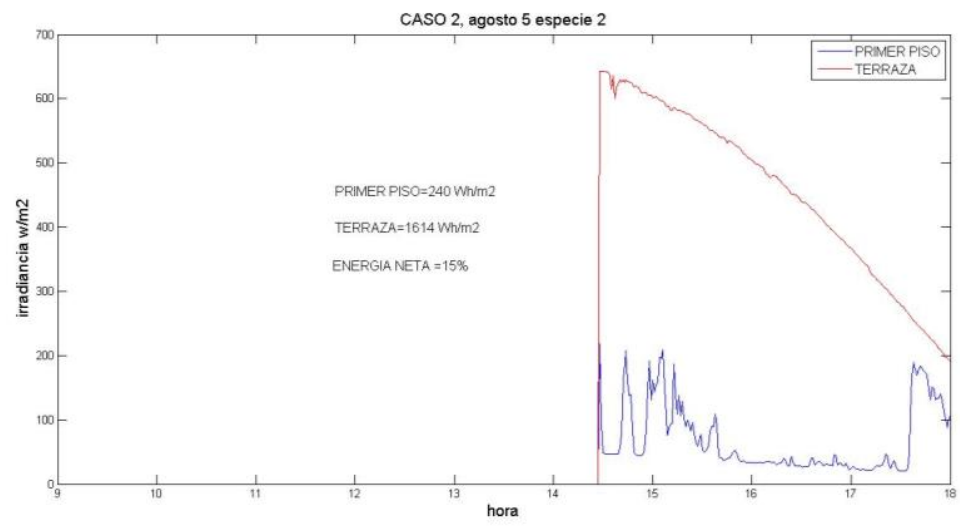

Fig. 16 Irradiancia medida en el plano vertical y orientado hacia el Norte a pleno asoleamiento y en condición de asoleamiento parcial

Fte: Elaboración propia 
Estos resultados muestran que el arbolado urbano resulta una variable de primer orden en la captación del recurso solar en entornos urbanos altamente forestados y deberían ser considerada la elección de la especie arbórea optima al momento de la planificación urbana y en la consideración de futuras estrategias de diseño.

\subsection{INFLUENCIA DE LA ALTURA DE LA EDIFICACION}

Para analizar el impacto de la morfología urbano-edilicia en función de la altura edilicia se han realizado mediciones desplazando verticalmente el sensor que mide la zona de fachada parcialmente asoleada. Fig. 17 a Fig. 19.

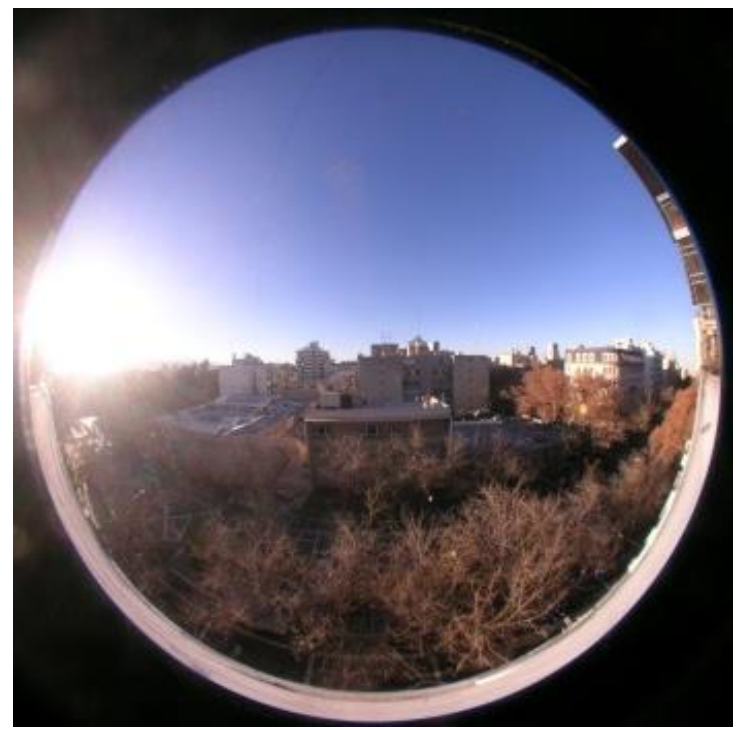

Fig. 17 Fotografía hemisférica del entorno tomada desde la ubicación del sensor. Zona de fachada con pleno asoleamiento. Terraza

Fte: Fotografía por Julieta A. Yamin Garretón

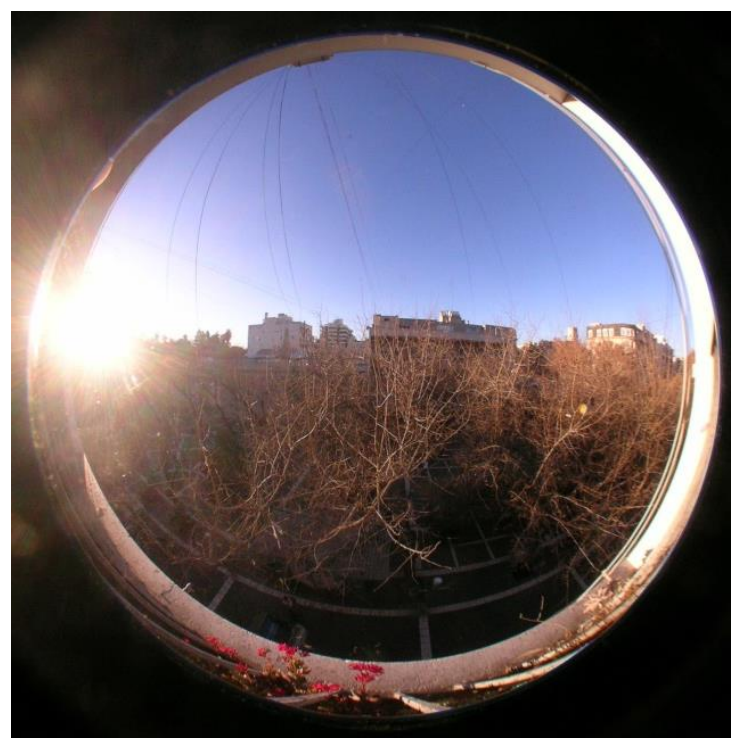

Fig. 18 Fotografía hemisférica del entorno tomada desde la ubicación del sensor. Caso 1. Zona de fachada parcialmente asoleada. Tercer piso.

Fte: Fotografía por Julieta A. Yamin Garretón 


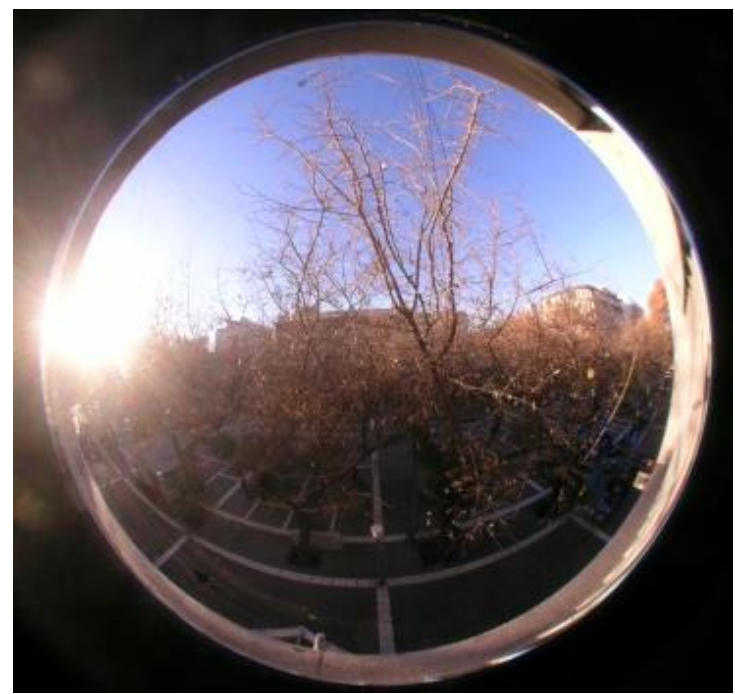

Fig. 19 Fotografía hemisférica del entorno tomada desde la ubicación del sensor. Caso 3. Zona de fachada parcialmente asoleada. Tercer piso.

Fte: Fotografía por Julieta A. Yamin Garretón

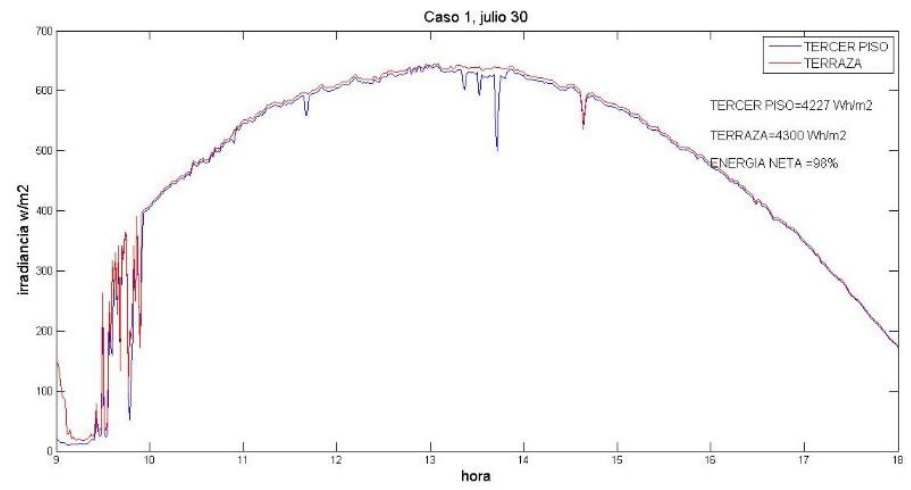

Fig. 20 Irradiancia medida en el plano vertical y orientado hacia el Norte a pleno asoleamiento y en condición de asoleamiento parcial

Fte: Elaboración propia

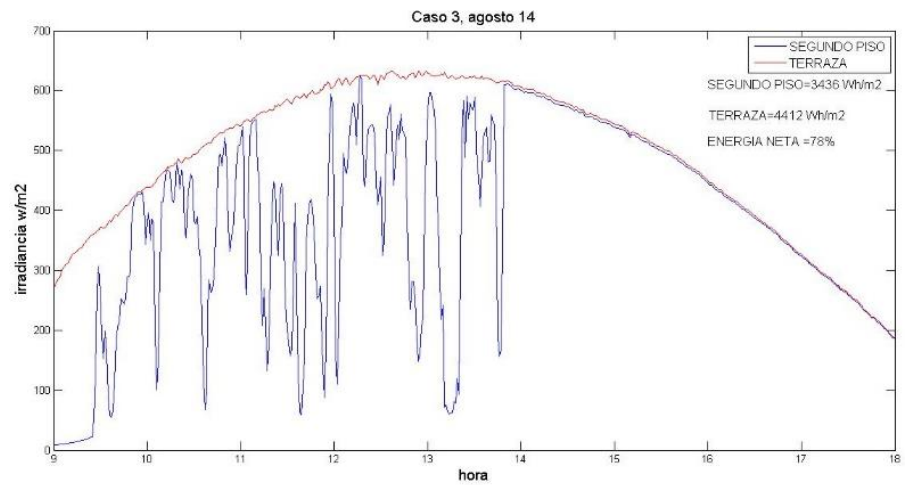

Fig. 21 Irradiancia medida en el plano vertical y orientado hacia el Norte a pleno asoleamiento y en condición de asoleamiento parcial

Fte: Elaboración propia 
Cuando el sensor que mide la zona parcialmente asoleada se ubicó en el tercer piso se tomaron valores que indican un $98 \%$ de la irradiancia total disponible, mientras que en la ubicación del segundo piso, los valores medidos indican una disponibilidad del $78 \%$ del recurso.

La gran diferencia entre el $2 \%$ y el $22 \%$ (Fig. 20 y Fig. 21) de enmascaramiento de la radiación solar, se puede explicar por el desarrollo máximo de la copa de los árboles presentes de dicha fachada norte que alcanzan una altura media de $10 \mathrm{mts}$ (Fig. 18 y Fig. 19). Estos datos medidos en una misma fachada moviendo el sensor en sentido vertical nos dan una información para elaborar estrategias que consideren las condiciones por sobre y por debajo de la copa de los arboles a fin de maximizar el uso del recurso solar disponible en los entornos urbanos.

La altura edilicia y los distintos niveles de construcción permiten definir situaciones deseadas de conservación y acceso pleno al recurso solar.
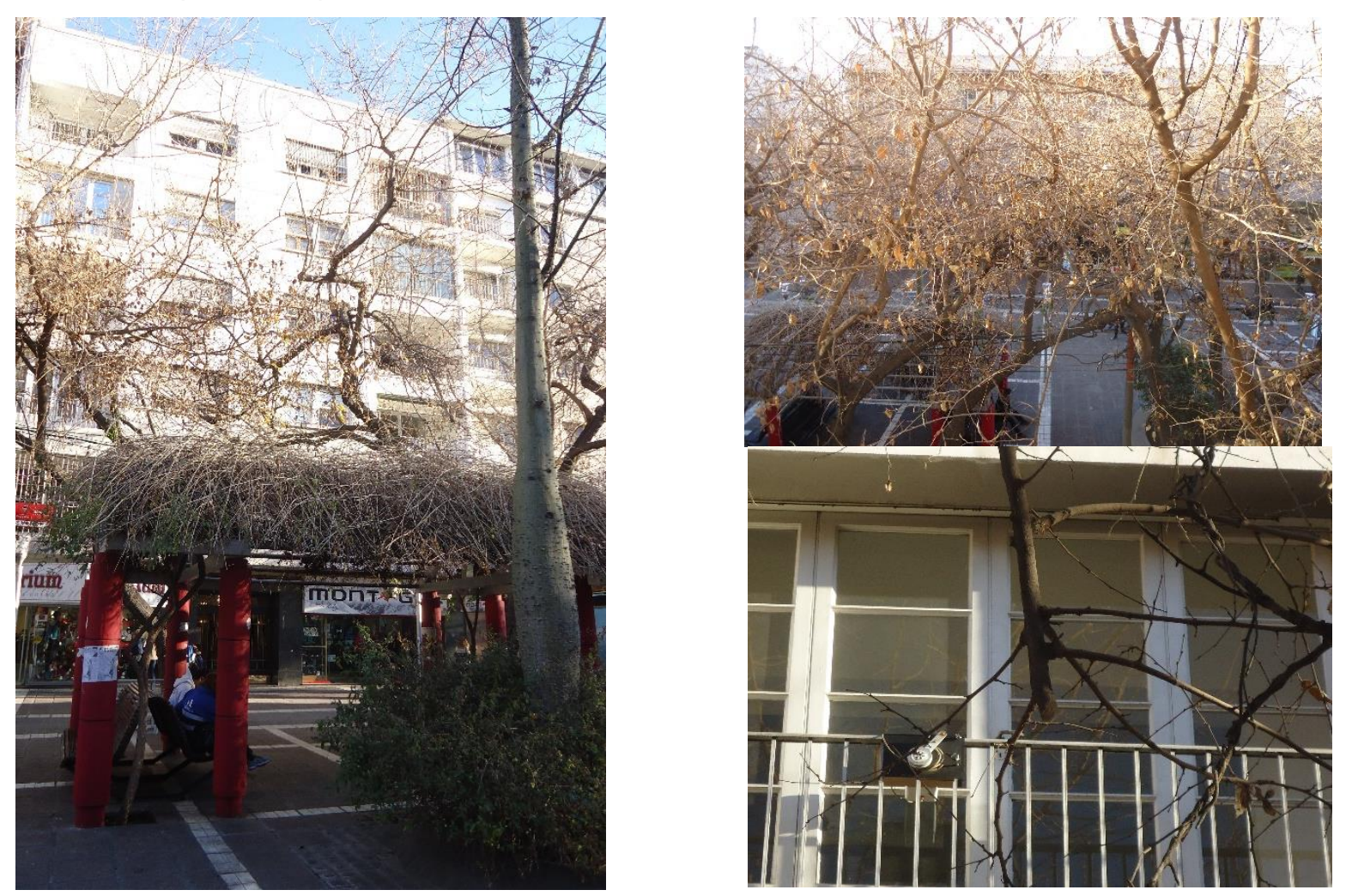

Fig. 22 Imágenes del Caso de Estudio 3 Fte: Elaboración propia

\section{Conclusiones}

Los resultados obtenidos evidencian el impacto de las principales variables que condicionan el acceso al sol en un entorno urbano altamente vegetado. Las once configuraciones urbanas estudiadas en el Área Metropolitana de Mendoza (AMM), presentan características diferenciales con respecto a variables urbanas y edilicias. En casi todos los casos el potencial solar de entornos urbanos residenciales presentan valores de irradiancia solar (W/m2) muy diferentes a las de pleno asoleamiento. Los valores medidos de Asoleamiento Efectivo en la estación de invierno fluctúan entre un máximo de $98 \%$ y un mínimo de $34 \%$, debido al enmascaramiento sólido y permeable (ramajes desnudos de los árboles). 
La importante influencia que presenta el ramaje desnudo en la estación invernal ha quedado demostrada. Estas características pueden acentuarse en ciertos forestales y bajo determinadas condiciones de posición solar, como lo muestran los valores medidos en el Caso de Estudio 2, donde en horas de la tarde se registró una disponibilidad del recurso del sólo un 15\%. Estos valores sugieren que hay que tener una consideración especial en la selección de especies que serán incluidas estructuralmente en entornos urbanos.

La morfología edilicia heterogénea condiciona fuertemente las posibilidades acceso al sol, esto ha quedado demostrado en el Caso de Estudio 6 en donde un edificio con entrantes y salientes produce un enmascaramiento sólido de hasta el $84 \%$ en horas de la mañana, en su propia fachada orientada al Norte. Cabe entonces recomendar la forma edilicia homogénea (que no produzca entrantes y salientes) para asegurar el asoleamiento pleno de las superficies potencialmente colectoras, tanto verticales al norte en conjuntos nuevos y en conjuntos existentes donde es una condición básica para el reciclado.

El desplazamiento vertical del sensor utilizado para medir irradiancia en la zona de fachada parcialmente asoleada demostró la importancia de establecer estrategias de diseño y legislación acorde a una ciudad altamente arbolada que genera condiciones distintas por sobre y por debajo del arbolado. También la influencia de la edificación circundante disminuye cuando consideramos alturas mayores en la edificación, esta combinación de efectos permitió medir una captación de la energía solar del 98\% de la condición de pleno asoleamiento en el Caso 1.

Los condicionantes urbanos para la captación de la energía solar en fachadas norte que hemos analizado (arbolado, morfología edilicia y altura de la edificación), se presentan con mayor intensidad cuando se combinan con un ancho de canal vial estrecho. Esta situación corresponde al Caso de Estudio 7 donde se producen los valores mínimos de radiación solar recibida considerando el horario completo de medición. Esto determina que las estrategias a seguir para cañones urbanos estrechos deben ser de mayor exigencia en cuanto al tipo de especies arbóreas permitidas, altura máxima de edificación, forma y retiros.

Los resultados alcanzados constituyen un aporte para contribuir a reformar y actualizar en forma progresiva los códigos urbanos y edilicios para implementar los máximos niveles de eficiencia energética y los mínimos impactos ambientales de las edificaciones urbanas.

\section{Bibliografía}

ARBOIT, M.; DIBLASI, A.; FERNÁNDEZ LLANO, J. C. AND DE ROSA, C. 2008. Assessing the solar potential of low density urban environments in andean cities with desert climates - The case of the city of Mendoza, in Argentina. Renewable Energy. Elsevier Editorial System. Volum. 33 Tomo 8, p.1733-1748. ISSN: 0960-1481. Renewable Energy. Oxford UK.

ARBOIT, M.; MESA, A DIBLASI, A.; FERNANDEZ LLANO, J. C. Y DE ROSA, C. 2010. Assessing the solar potential of low density urban environments in andean cities with desert climates - The case of the city of Mendoza, in Argentina. 2nd. Part. Renewable Energy. Elsevier Editorial System. Vol. 35 p.1551-1558 ISSN 0960-1481. UK.

BRAGER G, \& DE DEAR R. 2001. Climate, Comfort and Natural Ventilation: A new adaptive comfort standard For ASHRAE Standard 55. Proceedings of the Moving Thermal Comfort Standards into the 21st Century: 01-18.

CANTON,M.A.; FERNANDEZ,J.; CORTEGOSO,J.L.; DE ROSA,C. 2000. Environmental and energy impact of the urban forest in arid zone cities. Architectural Science Review, 44 (1) 3-16.

CAPELUTO, I.G.; YEZIORO, A.; SHAVIV, E. 2003. Climatic aspects in Urban Design. A Case study. Building and Environment, 38. 
CARDENAS, L.; MORALES, L.; RUIVO, C. 2012. Análisis del efecto del sombreamiento exterior en la radiación solar incidente sobre fachadas de un edificio educacional en un entorno urbano de alta densidad. Congreso Iberoamericano de Energía Solar.

DE ROSA, C. 1988. Low-cost Passive Solar Homes built in a Tempered Arid Climate. Thermal and Economic Evaluation. Proceedings of the 6th. International PLEA Conference:795-802.

JENKS, M.; BURTON, E.; WILLIAMS K. 1996. The Compact City. A Sustainable Urban Form?. E\&FN SPON.

OWENS S. 1986. Energy planning and urban form. London: Pion Ltd.

RED SOLARIMÉTRICA DE LA REPÚBLICA ARGENTINA.

SERVICIO METEOROLÓGICO NACIONAL DE LA REPÚBLICA ARGENTINA.

TOWN AND COUNTRY PLANNING ASSOCIATION. 1996. Planning for a sustainable environment. Ed. Andrew Blowers. Earthscan. 